\title{
Satisfação dos estudantes de educação a distância da UFSM: uma análise multifatorial'
}

Satisfaction of distance education student from UFSM: a multifactorial analysis

Kelmara Mendes Vieira², Franciele Ines Reis Kunkel ${ }^{3}$, Elena Maria Mallmann ${ }^{4}$

I Trabalho referente ao Edital n. I7/NTE/UAB - 2012

2,3,4 Universidade Federal de Santa Maria - UFSM, Santa Maria, RS

\begin{abstract}
Resumo
O presente estudo teve como objetivo avaliar os fatores determinantes da satisfação dos estudantes de educação a distância da Universidade Federal de Santa Maria mediante o desenvolvimento de um modelo de equações estruturais baseado em dados coletados em um survey que contemplou 416 estudantes. Os principais resultados descritivos sugerem que os estudantes estão satisfeitos com o sistema de aprendizagem, sua estrutura, o nível de dedicação de professores e tutores e com o curso de modo geral. O modelo proposto mostrou-se adequado para mensurar a satisfação geral dos estudantes, comprovando a influência positiva direta dos construtos flexibilidade e estrutura e organização do sistema e do curso e o impacto indireto dos fatores envolvimento do professor/tutor e interação. Os resultados do estudo fornecem feedback para a instituição avaliar os principais pontos positivos e negativos da estrutura dos cursos a distância, de modo a melhorar os níveis de satisfação e fortalecer a implementação do sistema de ensino a distância.
\end{abstract}

Palavras-chaves: satisfação dos estudantes, educação a distância, modelagem de equações estruturais.

\begin{abstract}
The present study aimed to evaluate the determinants of student satisfaction of distance education at the Federal University of Santa Maria through the development of a structural equation model based on data collected in a survey that included 416 students. The main descriptive results suggest that students are satisfied with the learning system, its structure, the level of dedication of teachers and tutors and the course in general. The proposed model was adequate to measure the overall satisfaction of students, confirming the positive influence of the constructs flexibility and system and course structure and organization and, the impact factors of indirect involvement of the teacher / tutor and interaction. The study results provide feedback to the institution assess the main strengths and weaknesses of the course structure in order to improve satisfaction levels and strengthen the implementation of the system of distance education
\end{abstract}

Keywords: learner satisfaction; distance education; structural modeling equation. 


\section{INTROdUÇÃo}

A utilização de tecnologias de rede no ambiente de ensino-aprendizagem trouxe significativas alterações para o sistema de ensino tornando-se um meio útil de instrução e provendo aos estudantes novas experiências de aprendizagem que antes não eram possíveis (SHER, 2009). Para Moore e Kearsley (2012), a Educação a Distância $(\mathrm{EaD})$ pode ser entendida como o processo planejado de ensino-aprendizagem onde o ensino ocorre em um lugar diferente da aprendizagem, ou seja, professores e estudantes estão separados espacialmente e temporalmente o que acaba por exigir a utilização de tecnologias especiais para a comunicação interativa. A educação a distância, segundo Liaw (2008), utiliza a tecnologia de rede para criar, promover, distribuir e facilitar a aprendizagem sem restrições de tempo ou lugar. Nesse sentido, possibilita aos estudantes receber instruções, apresentar trabalhos, interagir com colegas, professores e tutores a qualquer momento e em qualquer lugar (SHER, 2009). Tais peculiaridades da $\mathrm{EaD}$ tem promovido a democratização do ensino, haja visto que cada vez mais indivíduos, que antes não tinham condições de realizar um curso de nível superior, passaram a ter essa oportunidade devido à flexibilidade temporal e espacial proporcionada pelos cursos de EaD.

Nesse cenário de crescente desenvolvimento de cursos de educação a distância, a qualidade dos serviços oferecidos e o diagnóstico da satisfação dos discentes são fundamentais para as IES que buscam sobreviver no mercado educacional. A qualidade do curso, o envolvimento de professores/tutores e a satisfação dos acadêmicos com o curso, são fatores que determinam o perfil e o desempenho dos profissionais que ingressarão no mercado de trabalho. A formação de profissionais competentes é uma cobrança da sociedade. Walter, Tontini e Domingues (2005) afirmam que é imprescindível que as instituições de ensino não percam de foco a qualidade dos seus processos, bem como a qualidade da estrutura ofertada, devendo estar atentas às mudanças, identificando necessidades atuais e futuras da comunidade e transformando o processo de melhoria contínua em parte da cultura organizacional. Shee e Wang (2008) acrescentam que as IES além de enfatizar a qualidade de ensino, devem reconhecer os estudantes como peças chaves de seu sucesso e, por esse motivo, devem avaliar suas atitudes em relação ao sistema de aprendizado e ao curso como um todo. Essa avaliação, segundo os próprios autores, se justifica pelo fato de a satis- fação ser um fator crítico para a continuidade do uso do sistema de ensino online e o sucesso dos cursos de educação a distância.

A satisfação dos estudantes em relação à educação a distância pode ser compreendida, segundo Wang (2003), como uma resposta afetiva sumária de intensidade variável que acompanha atividades de aprendizagem online assíncronas e é estimulada por uma série de aspectos focais tais como conteúdo, interface do usuário, customização e desempenho da aprendizagem. O autor acrescenta que, por um lado, a satisfação do estudante de $\mathrm{EaD}$, assim como a satisfação de um cliente tradicional, refere-se à resposta afetiva decorrente de uma troca específica e ao julgamento avaliativo pós-compra. Por outro lado, refere-se a aspectos específicos do contexto da educação a distância. Para Lindgaard e Dudek (2003), a satisfação refere-se à soma subjetiva de experiências interativas, as quais são influenciadas por uma série de componentes afetivos ao longo da interação.

A complexidade envolvida na correta avaliação da satisfação dos estudantes de EaD (WANG, 2003; SUN et al.; 2008), estimula a realização de pesquisas que visem ao desenvolvimento de modelos de mensuração da satisfação (SHEE; WANG, 2008). A construção de modelos que incorporem diferentes aspectos da $\mathrm{EaD}$ se faz necessária, tendo em vista a multidimensionalidade da satisfação, a qual resulta de uma série de experiências interativas, influenciadas por elementos de natureza técnica e subjetiva do ambiente de EaD (LINDGAARD; DUDEK, 2003).

Para elaboração desse estudo buscou-se abranger, dentro do possível, várias dimensões propostas pela literatura como determinantes da satisfação a fim de construir um instrumento de diagnóstico adequado que permita a compreensão, fidedigna, do que realmente influencia no nível de satisfação dos estudantes (WANG, 2003). Dessa forma, o estudo contemplou a investigação dos fatores: envolvimento do professor/tutor, interação estudante/estudante, estudante/professor e estudante/tutor, flexibilidade, qualidade e adequação do conteúdo e estrutura e organização do sistema e do curso.

Paswan e Young (2002) definem envolvimento de professores e tutores como o nível de entusiasmo e interesse na promoção de um ambiente de ensino-aprendizagem online agradável e estimulante. Para Collis (1995), professores e tutores exercem papel crucial tanto na dinâmica do curso quanto na integração e motivação dos estudantes. Segundo Volery e Lord (2000), a superação das desvantagens da $\mathrm{EaD}$ como, por 
exemplo, o isolamento dos estudantes devido à ausência de um ambiente de sala de aula, passa pela capacidade dos professores e tutores em propor métodos e técnicas de ensino que promovam a motivação e a interação dos estudantes com seus colegas e com os próprios professores e tutores a fim de conduzir cursos de educação a distância bem sucedidos (YEE, 2011). A capacidade e o interesse de professores e tutores em solucionar os problemas, responder os questionamentos e atender as necessidades incentivam a continuidade da aprendizagem e influenciam diretamente no nível de satisfação dos estudantes (SUN et al., 2008).

Para Dziuban e Moskal (2001), a qualidade e a quantidade de interações são fatores críticos para a satisfação dos estudantes com o processo de aprendizagem e o sucesso dos cursos de EaD. Segundo Moore (1989), a interação estudante-estudante ocorre quando estudantes de diferentes regiões geográficas interagem uns com os outros através de grupos de discussão ou fóruns a fim de atingir determinado objetivo. Já as interações estudante-tutor e estudante-professor se verificam quando estudantes fazem questionamentos e participam das discussões propostas e, quando professores e tutores proporcionam o conhecimento do conteúdo, proveem as informações necessárias para o esclarecimento de dúvidas, fornecem feedback e melhoram a motivação dos estudantes. Um clima favorável de aprendizagem incentiva e estimula a troca de opiniões, informações e conhecimento, contribuindo para melhorar a satisfação do estudante com o curso (WU; TENNYSON; HSIA, 2010) e, inclusive, estimular o interesse, em termos de energia e recursos, dos professores e tutores pelo curso e pelos próprios estudantes (PASWAN; YOUNG, 2002).

A flexibilidade, conforme Sun et al. (2008), pode ser compreendida como a percepção dos estudantes no que se refere à eficiência e aos efeitos resultantes da escolha de um curso de modalidade a distância sobre o trabalho e a aprendizagem. Para Paechter, Maier e Macher (2010), a flexibilidade é visualizada como um incentivo na escolha da modalidade de ensino $\mathrm{EaD}$, por permitir um maior controle sobre o processo de aprendizagem, na medida em que os estudantes possuem liberdade para escolher o local, a hora e o ritmo de estudo (HONG; LAI; HOLTON, 2003). Essa vantagem, segundo Johnston, Killion e Oomen (2005), permite aos indivíduos aliar atividades pessoais ou profissionais corriqueiras com a realização de um curso em nível de graduação ou pós-graduação contribuindo para a maior participação e satisfação dos estudantes.
O fator qualidade e adequação do conteúdo diz respeito à quantidade e qualidade do conteúdo repassado, a validade do desenvolvimento de trabalhos, a adequação do tempo às tarefas e o nível das leituras indicadas pelo professor (PASWAN, YOUNG, 2002; OZKAN, KOSELER, 2009). O nível de qualidade do conteúdo, segundo Ozkan e Koseler (2009), está estreitamente relacionado à boa concepção e gestão do ambiente de aprendizagem. De acordo com os autores, os estudantes atribuem valor ao conteúdo quando o mesmo é bem organizado, efetivamente apresentado, interativo, claramente escrito, útil e flexível. A percepção quanto à atualidade e utilidade do conteúdo, atrelada à gestão eficaz do curso permite aos alunos sentirem-se mais confortáveis e seguros o que contribui para maiores taxas de permanência e satisfação com o curso (OZKAN; KOSELER, 2009). Para Yee (2011), tanto a efetiva qualidade do conteúdo quanto a percepção de que o conteúdo é comunicado de forma a facilitar a aprendizagem no ambiente de EaD são fatores críticos para a satisfação do estudante.

Conforme Paswan e Young (2002), a boa organização e estruturação do curso e do sistema de ensino, as quais abrangem às necessidades de aprendizagem, o uso adequado de estratégias pedagógicas, o estabelecimento de um ambiente presencial adequado e a utilização de um sistema online e de ferramentas de comunicação consistentes e confiáveis são determinantes para a melhor avaliação dos estudantes em relação a si próprios, aos professores e ao curso como um todo. A satisfação dos estudantes com o ambiente melhora a percepção quanto à adequação da estrutura e da tecnologia utilizada no curso promovendo a maior participação no processo de aprendizagem (LIAW, 2008). O Quadro 1 apresenta um resumo dos fatores investigados e da relação esperada entre eles.

Em meio a tais reflexões, o objetivo geral desse trabalho foi propor e validar um modelo de satisfação dos estudantes de ensino a distância da UFSM. Especificamente buscou-se, i) avaliar o nível de satisfação dos estudantes com os cursos de educação a distância, ii) avaliar a percepção dos estudantes quanto aos construtos envolvimento do tutor/professor, interação, qualidade e adequação do conteúdo, flexibilidade e organização e estrutura do sistema e do curso, iii) identificar e validar cada um dos construtos propostos e iv) analisar a influência direta e indireta dos construtos na satisfação. Para a consecução dos objetivos, foram aplicados questionários a 416 estudantes de 15 cursos de EaD da UFSM.

Como pode ser constatado nas publicações 


\begin{tabular}{|c|c|c|c|c|}
\hline Hipótese & $\begin{array}{c}\text { Variáveis } \\
\text { independentes }\end{array}$ & $\begin{array}{c}\text { Variáveis } \\
\text { dependentes }\end{array}$ & $\begin{array}{l}\text { Relação } \\
\text { esperada }\end{array}$ & Autores \\
\hline H1 & $\begin{array}{l}\text { Envolvimento } \\
\text { do } \\
\text { professor/tutor }\end{array}$ & $\begin{array}{l}\text { Satisfação dos } \\
\text { estudantes de } \\
\text { EaD }\end{array}$ & Positiva & $\begin{array}{l}\text { Inman, Kervin e Mayes (1999), Sun et al } \\
\text { (2008), Paechter, Maier e Macher (2010) }\end{array}$ \\
\hline $\mathrm{H} 2$ & \multirow{2}{*}{ Interação } & $\begin{array}{l}\text { Satisfação dos } \\
\text { estudantes de } \\
\text { EaD }\end{array}$ & Positiva & $\begin{array}{l}\text { Valentaet al. (2001), Wu, Tennyson e Hsia } \\
\text { (2010), Chou (2003), Sher (2009) }\end{array}$ \\
\hline $\mathrm{H} 3$ & & $\begin{array}{l}\text { Envolvimento } \\
\text { do } \\
\text { professor/tutor }\end{array}$ & Positiva & $\begin{array}{c}\text { Paswan e Young (2002), Vieira, Kunkel e } \\
\text { Righi (2012) }\end{array}$ \\
\hline $\mathrm{H} 4$ & Flexibilidade & $\begin{array}{l}\text { Satisfação dos } \\
\text { estudantes de } \\
\text { EaD }\end{array}$ & Positiva & $\begin{array}{l}\text { Johnston, Killion e Oomen(2005), Sun et al. } \\
\text { (2008), }\end{array}$ \\
\hline H5 & $\begin{array}{l}\text { Qualidade e } \\
\text { adequação do } \\
\text { conteúdo }\end{array}$ & $\begin{array}{l}\text { Satisfação dos } \\
\text { estudantes de } \\
\text { EaD }\end{array}$ & Positiva & $\begin{array}{c}\text { Inman, Kervin e Mayes (1999), Paswan e } \\
\text { Young (2002), Ozkan e Koseler (2009), Yee } \\
\text { (2011) }\end{array}$ \\
\hline H6 & \multirow{4}{*}{$\begin{array}{c}\text { Estrutura e } \\
\text { organização do } \\
\text { curso }\end{array}$} & $\begin{array}{l}\text { Satisfação dos } \\
\text { estudantes de } \\
\text { EaD }\end{array}$ & Positiva & $\begin{array}{l}\text { Piccoli et al. (2001), Paswan e Young } \\
\text { (2002), Macdonald e Thompson (2005) }\end{array}$ \\
\hline $\mathrm{H} 7$ & & $\begin{array}{l}\text { Qualidade e } \\
\text { adequação do } \\
\text { conteúdo }\end{array}$ & Positiva & $\begin{array}{l}\text { Pituch e Lee (2006), Wu, Tennyson e Hsia } \\
\text { (2010) }\end{array}$ \\
\hline $\mathrm{H} 8$ & & Interação & Positiva & $\begin{array}{l}\text { Pituch e Lee (2006), Wu, Tennyson e Hsia } \\
\text { (2010) }\end{array}$ \\
\hline H9 & & Flexibilidade & Positiva & Pituch e Lee (2006) \\
\hline
\end{tabular}

Quadro 1: resumo dos fatores investigados e da relação esperada entre eles.

Fonte: Elaborado pelos autores, 2013.

nacionais e internacionais, a maioria das pesquisas sobre satisfação dos estudantes de cursos de EaD emprega métodos estatísticos que possibilitam examinar apenas uma única relação de cada vez, como a correlação, a regressão múltipla e análises de variância (SUN et al., 2008). Nesse estudo, optou-se pelo emprego da técnica de Modelagem de Equações Estruturais por possibilitar ao pesquisador testar um número maior de relações entre as variáveis e os construtos em análise, sendo, portanto, um modelo mais robusto para mensurar o construto satisfação.

Entre as principais contribuições do estudo, ressaltam-se: i) a compreensão dos fatores que afetam a satisfação dos estudantes com os cursos de $\mathrm{EaD}$ o que possibilita o desenvolvimento de ações, por parte da instituição de ensino superior, que visem melhor estruturar os pontos mais valorizados pelos estudantes e, ii) o avanço da literatura nacional à medida que se investiga o impacto tanto de fatores técnicos quanto subjetivos sobre a satisfação dos estudantes. Este trabalho está dividido em cinco seções, incluindo a introdução. A segunda seção apresenta a base teórica e empírica que sustenta o estudo. A terceira parte contempla os procedimentos metodológicos adotados. $\mathrm{Na}$ sequência, são apresentados os resultados e, por fim, as considerações mais relevantes sobre a temática abordada, as limitações e as principais sugestões para estudos futuros.

\section{Método}

Visando atingir o objetivo proposto, realizou-se uma pesquisa descritiva de cunho quantitativo. O estudo considerou como universo de pesquisa os estudantes de cursos da modalidade de EaD da Universidade Federal de Santa Maria (UFSM). A Universidade conta com 4.372 estudantes matriculados, os quais estão distribuídos em 41 polos, localizados nos estados do Rio Grande do Sul (36), Paraná (2), São Paulo (1), Tocantins (1) e Ceará (1).

Os questionários, aplicados através do sistema Moodle (sistema de gerenciamento de cursos a distância utilizado pela UFSM), foram disponibilizados aos estudantes durante os meses de outubro, novembro e dezembro. Para realização da pesquisa, criou-se uma disciplina, denominada Avaliação do Curso, a qual "hospedou" o 


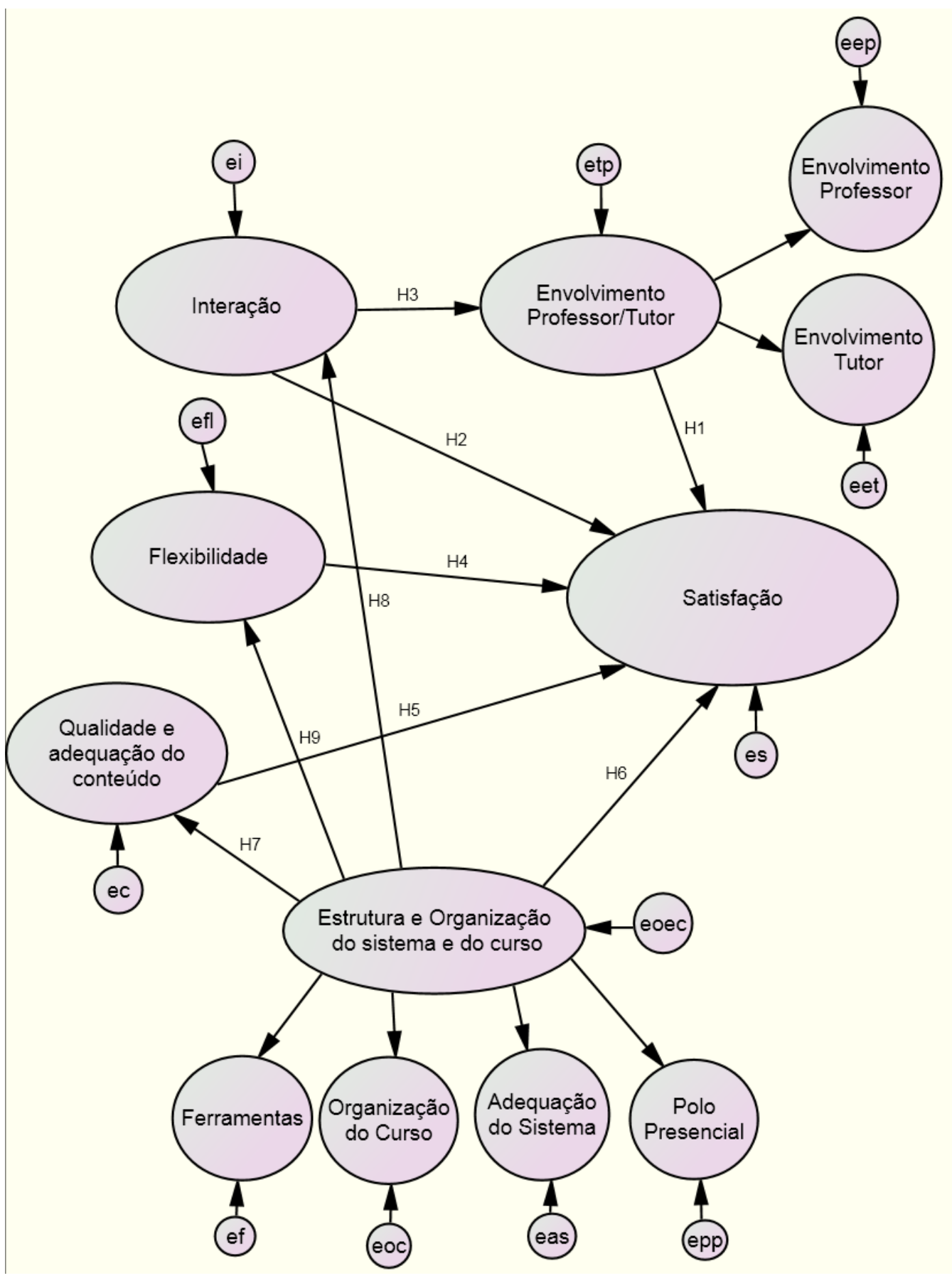

Imagem 1

Fonte: Elaborado pelos autores, 2013.

questionário. Foram disparados e-mails, em duas oportunidades, a todos os estudantes matriculados a fim de divulgar a realização da pesquisa. A amostra final foi composta por 470 estudantes. Do total de questionários acessados, 54 tiveram de ser excluídos por estarem preenchidos de forma incompleta, perfazendo uma amostra total de 416 respondentes. É importante destacar que esse tamanho de amostra é superior ao mínimo recomendado que é de cinco respondentes por indicador (HAIR et al., 2009).

$\mathrm{O}$ instrumento de coleta de dados foi composto por doze blocos de perguntas. $\mathrm{O}$ primeiro e o segundo bloco buscaram investigar o nível de envolvimento e interesse dos professores e tutores, respectivamente. As questões referentes ao envolvimento dos professores, em número de 8 , foram adaptadas de Ozkan e Koseler (2009). Já as ques- 
tões referentes ao envolvimento dos tutores foram elaboradas com base nos trabalhos de Giarola et al. (2009) e Oliveira et al. (2012) perfazendo um total de 8 questões. Para avaliar a percepção dos estudantes quanto à efetividade de sua interação com colegas, professores e tutores foram utilizadas 6 questões adaptadas de Sun et al. (2008). Na quarta parte procurou-se identificar, através de uma versão adaptada da escala de flexibilidade percebida de Sun et al. (2008), a percepção dos estudantes quanto às vantagens ocasionadas pela flexibilidade temporal e espacial decorrentes da realização de um curso a distância. Para mensurar a qualidade e adequação do conteúdo foram utilizadas 11 questões elaboradas com base nas escalas propostas por Paswan e Young (2002) e Ozkan e Koseler (2009).

No sexto bloco buscou-se verificar a percepção dos respondentes no que se refere à adequação do sistema. Para tanto foram utilizadas 5 questões adaptadas de Ozkan e Koseler (2009). O fator ferramentas de comunicação, que busca avaliar a utilidade dos meios de comunicação, foi mensurado com base na escala proposta por Oliveira et al. (2012). Para avaliar o fator polo presencial, o qual indica o nível de estruturação e organização do ambiente presencial, foi empregada a escala proposta por Oliveira et al. (2012), a qual é formada por 8 questões. O fator organização do curso foi mensurado com base nas questões propostas por Paswan e Young (2002). Para avaliar a satisfação dos estudantes com o curso de EaD foram utilizadas 8 questões elaboradas a partir das escalas propostas por Paswan e Young (2002) e Sun et al. (2008). Todos os fatores acima mencionados foram avaliados através de uma escala do tipo likert de 5 pontos, onde o primeiro ponto representava total discordância e o quinto ponto total concordância com as afirmações feitas. O último bloco contém dez questões que visaram identificar o perfil dos respondentes, através de aspectos como gênero, idade, estado civil, curso, polo, entre outros. O questionário aplicado encontra-se no Apêndice 1.

Ressalta-se que o questionário foi enviado para especialistas da área de $\mathrm{EaD}$ para verificação de sua confiabilidade e validade. Seguindo sugestão dos especialistas, alguns itens foram revistos e outros excluídos a fim de facilitar o entendimento por parte dos respondentes. Para a análise dos dados foram utilizados dois softwares: SPSS 18.0® e Amos ${ }^{\mathrm{TM}}$. Em um primeiro momento, realizou-se a estatística descritiva dos dados com o objetivo de avaliar cada indicador separadamente. Na sequência, realizaram-se testes de diferença de média, teste t e Anova, visando verificar se há diferença na avaliação de cada um dos fatores investigados se considerado o perfil do respondente.

Com vistas a verificar a influência dos fatores: envolvimento do professor/tutor, interação, flexibilidade, qualidade e adequação do conteúdo e estrutura e organização do sistema e do curso sobre a satisfação do estudante aplicou-se a Modelagem de Equações Estruturais (MEE) que, conforme Hair et al. (2009), possibilita a verificação simultânea de uma série de relações de dependência ao combinar aspectos da regressão múltipla e da análise fatorial. A Figura 1 apresenta o modelo conceitual testado nesse estudo.

A avaliação do modelo foi realizada em duas etapas, conforme sugestão de Anderson e Gerbing (1988). Inicialmente realizou-se uma Análise Fatorial Confirmatória (AFC) para validar os construtos. Os relacionamentos entre as variáveis observadas e seus construtos foram estimados utilizando o método da máxima verossimilhança. Na segunda etapa, o modelo híbrido foi validado através dos índices de ajuste do modelo global e da significância e magnitude dos coeficientes das regressões estimadas. Seguindo a recomendação de Anderson e Gerbing (1988), a validade do modelo foi avaliada através da veri $\square$ cação da validade convergente, unidimensionalidade e confiabilidade dos construtos.

A validade convergente de cada construto foi analisada pela observação da magnitude e signii $\square$ cância estatística dos coe $\square$ cientes padronizados e pelos índices de ajuste absolutos: estatística qui-quadrado $\left(\square^{2}\right)$, root mean squares residual $(\mathrm{RMR},<0,10)$, root mean square error of aproximation (RMSEA, < 0,08), goodness-of-fit index (GFI, varia de 0 a 1 , onde 1 indica perfeito ajustamento) e índices de ajuste comparativos: comparative fit index (CFI, $>0,9)$, normed fit index (NFI, $>0,9$ ), tucker-lewis index (TLI, >0,9) (GARVER, MENTZER, 1999; HAIR et al., 2009). Para mensurar a confiabilidade dos construtos utilizou-se o Alfa de Cronbach e a Variância Média Extraída que, segundo Hair et al. (2009), devem possuir valores superiores a 0,7 e 0,5 , respectivamente. A veri $\square$ cação da unidimensionalidade do construto foi realizada mediante avaliação dos resíduos padronizados. Nesse procedimento, foram considerados unidimensionais os construtos que apresentaram para todos os pares formados por variáveis observadas, resíduos padronizados inferiores a 2,58 (HAIR et al., 2009).

Para a análise do modelo integrado, que agrega o modelo de mensuração e o modelo estrutural, optou-se pela estratégia de aprimoramento, na qual, a partir de um modelo inicialmente pro- 
posto são feitas modificações para se chegar a um modelo ajustado. No procedimento de ajuste, os coeficientes de regressão não significativos foram retirados, sendo incorporadas covariâncias não previstas inicialmente.

\section{Análise e discussão dos resultados}

A pesquisa compreendeu os estudantes dos cursos da modalidade de EaDda Universidade Federal de Santa Maria, sendo a amostra final composta por 416 estudantes. Quanto ao perfil, constatou-se que a maior parcela é do sexo feminino $(81, \%)$, casado $(46,39 \%)$ e possui, em média, 35 anos. No que se refere ao número de horas semanais dedicadas aos estudos, os respondentes alegaram que, em média, destinam 14 horas de sua semana aos estudos. Quanto ao semestre que estão cursando, verificou-se que a maior parcela dos respondentes concentra-se nos três primeiros períodos $(52,4 \%)$. Entretanto, um bom número de respondentes $(36,76 \%)$ cursa um dos 3 últimos semestres - sexto, sétimo e oitavo semestre. Tal distribuição diminui a possibilidade de obtenção de resultados viesados.

Participaram da pesquisa estudantes de 8 cursos de graduação e 7 cursos de especialização, sendo que os cursos de maior representatividade amostral foram: Licenciatura em Pedagogia $(22,4 \%)$, Bacharelado em Administração Pública $(13,4 \%)$, Licenciatura em Letras Português e Literatura (8,61\%), Licenciatura em Letras Espanhol e Literatura (8,37\%), Licenciatura em Sociologia $(7,17 \%)$ e Especialização em Educação Ambiental
$(5,26 \%)$. Os cursos que tiveram menor representatividade amostral foram Licenciatura em Matemática (0,24\%), Especialização em Gestão Pública Municipal (1,67\%), Especialização em Gestão Pública (2,15\%) e Licenciatura em Física $(2,63 \%)$. Tal resultado pode ser explicado pelo menor número de estudantes matriculados nesses cursos ou pelo menor interesse dos mesmos em participar da pesquisa.

Quanto ao polo, verificou-se que os respondentes estão distribuídos em 38 polos, sendo que os de maior participação foram: Sobradinho $(10,81 \%)$, Restinga Seca $(9,61 \%)$, Três Passos (9,17\%), Tio Hugo (6,97\%), Santana do Livramento (6,73\%), Três de Maio (3,84\%) e São Lourenço do Sul $(3,84 \%)$. Diversos polos apresentaram número muito pequeno de participantes, como por exemplo, Balneário Pinhal, Cerro Largo, Constantina, Jacuizinho, Santa Maria, Santo Antônio da Patrulha e Sapiranga, os quais tiveram a participação de somente 2 respondentes por polo.

Visando avaliar a opinião dos respondentes em relação aos construtos pesquisados, procedeu-se ao cálculo da média, mediana e desvio padrão de cada construto como pode ser visualizado na Tabela 1.

Todos os construtos foram calculados com base na pontuação média obtida a partir das respostas individuais dos estudantes para cada questão componente do construto. A obtenção de um único valor representativo do construto simplifica a realização de testes entre os construtos e facilita o entendimento do leitor (SHER, 2009).

Considerando que as escalas variaram de 1 a 5 , onde 1 indicava discordância total e 5 concordância total, verificou-se que os estudantes

Tabela 1 - Média, mediana e desvio-padrão dos fatores

\begin{tabular}{lccc}
\hline \multicolumn{1}{c}{ Fatores } & Média & Mediana & $\begin{array}{c}\text { Desvio } \\
\text { padrão }\end{array}$ \\
Envolvimento do professor & 3,707 & 4,000 & 0,698 \\
Envolvimento do tutor & 3,880 & 4,000 & 0,709 \\
Interação & 3,720 & 4,000 & 0,531 \\
Flexibilidade & 3,840 & 4,000 & 0,650 \\
Qualidade e adequação do & 3,907 & 4,000 & 0,680 \\
conteúdo & 3,816 & 4,000 & 0,680 \\
Ferramentas de comunicação & 3,723 & 4,000 & 0,669 \\
Adequação do sistema & 3,946 & 4,000 & 0,677 \\
Organização do curso & 3,880 & 4,000 & 0,690 \\
Polo presencial & 4,363 & 4,500 & 0,622 \\
Satisfação & & & \\
\hline
\end{tabular}

Fonte: Elaborado pelo autor, 2013. 
tem uma percepção positiva em relação a todos os fatores investigados, se observado que as médias foram superiores a 3,7 e as medianas, em sua maioria, iguais a 4 . O fator mais bem avaliado foi a satisfação $(4,363)$, o que demonstra que os estudantes tem uma percepção favorável quanto ao atendimento de suas expectativas e necessidades fazendo com que os estudantes queiram retribuir aos benefícios recebidos, contribuindo com o curso após sua conclusão e recomendando o mesmo a pessoas conhecidas. Esse resultado vem a corroborar os achados de Hong, Lai e Holton (2003), Liaw (2008), Sun et al. (2008) e Sher (2009) que também observaram que os estudantes estão satisfeitos com a experiência de aprendizagem online. Além disso, os estudantes avaliaram positivamente o nível de organização do curso $(3,94)$ e o nível de qualidade e adequação do conteúdo $(3,90)$.

Os construtos envolvimento do tutor $(3,88)$ e do professor $(3,70)$ receberam, igualmente, avaliação positiva dos respondentes indicando que professores e tutores demonstram entusiasmo e interesse na promoção de um ambiente de ensino-aprendizagem online agradável e estimulante (PASWAN; YOUNG, 2002). Confirmando os achados de Liaw (2008) e Sun et al. (2008), observou-se que os estudantes apreciam a flexibilidade $(3,84)$ temporal e espacial proporcionada pelo curso e julgam adequados as ferramentas de comunicação
$(3,81)$ e o sistema base $(3,72)$ utilizados para manter a comunicação e a interatividade. A interação estudante/estudante, estudante/professor e estudante/tutor também foi considerada satisfatória. De modo geral, o baixo desvio-padrão apresentado por todos os fatores indica homogeneidade de respostas.

Visando verificar possíveis diferenças de média entre os grupos quanto aos fatores investigados foram realizados testes paramétricos de diferença de média. Para a análise da diferença de média entre dois grupos aplicou-se o Teste $t$ (1) e para mais de dois grupos utilizou-se o teste F (2). Os resultados estão expostos na Tabela 2.

Observando os resultados, verificou-se que não há diferença estatisticamente significativa entre os respondentes quanto aos fatores: interação, flexibilidade, ferramentas de comunicação e polo presencial o que indica, para esse estudo, que todos os respondentes avaliam de forma positiva esses construtos. Quanto aos fatores envolvimento de professores e tutores, adequação do sistema e organização do curso, constatou-se que os estudantes apresentam diferentes percepções se considerado o curso. Para todos esses fatores, observou-se que o maior grau de concordância e satisfação é apresentado pelos estudantes de cursos classificados na categoria Multidisciplinar, a qual abrangeu os cursos de Educação Ambiental, Educação Espe-

Tabela 2 - Valor e Significância dos Testes t (1) e Teste F (2) para os fatores envolvimento do professor, envolvimento do tutor, interação, flexibilidade, qualidade e adequação do conteúdo, ferramentas de comunicação, adequação do sistema, organização do curso, polo presencial e satisfação

\begin{tabular}{|c|c|c|c|c|c|c|c|c|c|c|}
\hline \multirow{2}{*}{ Fatores/Grupos } & \multicolumn{2}{|c|}{ Gênero (1) } & \multicolumn{2}{|c|}{ Estado civil (2) } & \multicolumn{2}{|c|}{$\operatorname{Idade}^{1}(2)$} & \multicolumn{2}{|c|}{$\begin{array}{l}\text { Horas de } \\
\text { estudo (2) }\end{array}$} & \multicolumn{2}{|c|}{$\operatorname{Curso}^{2}(2)$} \\
\hline & Valor & Sig & Valor & Sig & Valor & Sig & Valor & Sig & Valor & Sig \\
\hline Envolvimento do professor & 0,970 & 0,332 & 0,538 & 0,585 & 0,973 & 0,405 & 0,553 & 0,646 & 5,434 & $0,001 *$ \\
\hline Envolvimento do tutor & 0,173 & 0,863 & 0,678 & 0,508 & 1,402 & 0,405 & 0,271 & 0,846 & 2,869 & $0,037 * *$ \\
\hline Interação & 0,847 & 0,398 & 0,104 & 0,901 & 1,667 & 0,173 & 2,402 & 0,067 & 0,917 & 0,433 \\
\hline Flexibilidade & 0,959 & 0,338 & 1,299 & 0,274 & 2,004 & 0,119 & 1,378 & 0,249 & 1,203 & 0,309 \\
\hline Qualidade e adequação do conteúdo & 0,187 & 0,852 & 1,530 & 0,218 & 2,632 & $0,050 * *$ & 1,427 & 0,234 & 2,256 & 0,082 \\
\hline Ferramentas de comunicação & 0,067 & 0,852 & 1,381 & 0,253 & 1,027 & 0,380 & 0,793 & 0,498 & 1,047 & 0,372 \\
\hline Adequação do sistema & 0,500 & 0,996 & 0,012 & 0,988 & 1,346 & 0,259 & 0,677 & 0,566 & 2,932 & $0,034^{* *}$ \\
\hline Organização do curso & 0,619 & 0,537 & 1,598 & 0,203 & 1,499 & 0,214 & 1,207 & 0,307 & 2,758 & $0,043 * *$ \\
\hline Polo presencial & 0,931 & 0,353 & 2,746 & 0,065 & 1,591 & 0,191 & 0,363 & 0,780 & 1,533 & 0,206 \\
\hline Satisfação & 0,626 & 0,532 & 5,885 & $0,003 * *$ & 3,770 & $0,011^{* *}$ & 3,344 & 0,019 & 1,603 & 0,189 \\
\hline
\end{tabular}

Fonte: Elaborado pelos autores, 2013.

*significativo a $1 \% ;{ }^{* *}$ significativo a $5 \%$.

\footnotetext{
*Notas: ${ }^{1}$ Para transformar a idade em uma variável ordinal, a mesma foi dividida em quartis de modo que cada opção contivesse $25 \%$ das observações. A partir desse procedimento, consideraram-se os estudantes com até 27 anos, de 27 a 32 anos, de 33 a 40 anos e acima de 40 anos.

${ }^{2}$ Em virtude de vários cursos terem apresentado poucos respondentes, optou-se por reuni-los conforme a área a qual estão vinculados. Para proceder a tal categorização, utilizou-se por base a classificação da Coordenação de Aperfeiçoamento de Pessoal de Nível Superior (CAPES).
} 
cial e Gestão Educacional. Tal resultado sugere que os estudantes de tais cursos acreditam que, i) os professores e tutores dominam o conteúdo, estimulam a participação e incentivam a interação entre os estudantes, ii) o sistema Moodle é adequado e suporta a interatividade via chats, blogs e fóruns e, iii) o curso, de modo geral, apresenta boa infraestrutura e organização. Considerando ainda a diferença de percepção entre os cursos, chama a atenção o fato de a menor média, em todos os construtos, ter sido apresentada por estudantes da área de Linguística, Letras e Artes a qual englobou os cursos de Licenciatura em Letras Português e Literatura e Licenciatura em Letras Espanhol e Literatura indicando menor satisfação desses estudantes se comparado aos demais.

Para o fator qualidade e adequação do conteúdo houve diferença de média somente para a variável idade. Os estudantes com mais de 40 anos, se comparados aos demais, tem uma percepção mais favorável quanto à adequação do conteúdo, seu nível de qualidade e atualidade. No que se refere ao fator satisfação, foram encontradas diferenças significativas quanto ao estado civil e a idade. Indivíduos casados e com mais de 40 anos apresentam maiores níveis de satisfação com o curso.

\section{I Validação individual dos construtos}

Na primeira etapa da Modelagem de Equações Estruturais, buscou-se validar o modelo de mensuração, ou seja, cada um dos construtos estabelecidos inicialmente. Para a validação desses construtos foi realizada a AFC, sendo que os relacionamentos entre as variáveis observadas e os seus construtos foram estimados utilizando o método da máxima verossimilhança.
Após o cálculo inicial dos índices de ajuste e dos índices de confiabilidade, verificou-se que os construtos organização do curso, adequação do sistema e ferramentas de comunicação apresentaram valores dentro dos padrões recomendados pela literatura, fazendo com os mesmos mantivessem sua formação original. Os demais construtos não apresentaram índices adequados o que exigiu a realização de modificações a fim de ajustar o modelo. Entre as modificações realizadas houve a exclusão de variáveis cuja carga fatorial era inferior a 0,5 e o estabelecimento de correlações entre os erros das questões, conforme sugestão do pacote estatístico. Apesar de sugerido pelo software, somente foram correlacionados os erros para os quais houve aparato teórico que justificasse tal procedimento. O Quadro 2 apresenta todas as modificações realizadas para cada um dos construtos.

Após a realização das alterações citadas no Quadro 1, todos os construtos passaram a apresentar i) valor de qui-quadrado não significativo, ii) validade convergente, tendo em vista que os coeficientes padronizados apresentaram carga fatorial superior a 0,5 e foram significativos, os índices CFI, GFI, NFI e TLI foram superiores a 0,9 e os índices RMR e RMSEA foram inferiores a 0,10 e 0,08 , respectivamente, iii) confiabilidade, pois o Alpha de Cronbach e a Variância Média Extraída foram superiores ao valor mínimo de 0,70 e 0,50 , respectivamente e, iv) unidimensionalidade, dado que o valor de todos os resíduos padronizados foi inferior a 2,58 a um nível de significância de 5\% (ANDERSON, GERBING, 1998; GARVER, MENTZER, 1999; HAIR et al., 2009). Os resultados obtidos em relação aos índices de ajuste podem ser visualizados na Tabela 3 .

\subsection{Validação do modelo integral}

\begin{tabular}{|c|c|c|}
\hline \multirow[b]{2}{*}{ Construtos } & \multicolumn{2}{|c|}{ Procedimentos de ajuste do modelo } \\
\hline & $\begin{array}{l}\text { Exclusão de variáveis com carga } \\
\text { fatorial inferior a } 0,5\end{array}$ & $\begin{array}{l}\text { Estabelecimento de correlações } \\
\text { entre os erros das questões }\end{array}$ \\
\hline Envolvimento do professor & Q10 $(0,447)$ & $\begin{array}{c}\mathrm{eQ} 12 \leftrightarrow \mathrm{eQ} 13 ; \\
\mathrm{eQ} 12 \leftrightarrow \mathrm{eQ} 15 ; \mathrm{eQ} 13 \leftrightarrow \mathrm{eQ} 15\end{array}$ \\
\hline Envolvimento do tutor & - & $\mathrm{eQ} 4 \leftrightarrow \mathrm{eQ} 7$ \\
\hline Interação & Q22 $(0,069) ;$ Q17 $(0,285) ;$ Q21 $(0,337)$ & - \\
\hline Flexibilidade & Q70 $(0,274) ;$ Q69 $(0,380) ;$ Q67 $(0,380)$ & - \\
\hline $\begin{array}{l}\text { Qualidade e adequação do } \\
\text { conteúdo }\end{array}$ & Q41 $(0,496)$ & $\begin{array}{c}\mathrm{eQ} 40 \leftrightarrow \mathrm{eQ} 32 ; \mathrm{eQ} 39 \leftrightarrow \mathrm{eQ} 36 ; \\
\mathrm{eQ} 35 \leftrightarrow \mathrm{eQ} 34\end{array}$ \\
\hline Polo presencial & Q48 $(0,446)$, Q47 $(0,446) ;$ Q51 $(0,461)$ & $\mathrm{eQ} 49 \leftrightarrow \mathrm{eQ} 50 ; \mathrm{eQ} 50 \leftrightarrow \mathrm{eQ} 52$ \\
\hline Satisfação & Q60 (0,429); Q59 $(0,559)$ & $\begin{array}{l}\mathrm{eQ} 57 \leftrightarrow \mathrm{eQ} 56 ; \mathrm{eQ} 62 \leftrightarrow \mathrm{eQ} 61 \\
\mathrm{eQ62} \leftrightarrow \mathrm{eQ} 57 ; \mathrm{eQ} 57 \leftrightarrow \mathrm{eQ} 58\end{array}$ \\
\hline
\end{tabular}

Quadro 2 - Procedimentos utilizados para ajustar os modelos de mensuração

Fonte: elaborado pelos autores, 2013. 
Tabela 3 - Resultados dos índices de ajuste

\begin{tabular}{lccccc}
\hline \multicolumn{1}{c}{ Índices de ajuste } & $\begin{array}{c}\text { Envolvimento } \\
\text { do professor }\end{array}$ & $\begin{array}{c}\text { Envolvimento } \\
\text { do tutor }\end{array}$ & Interação & Flexibilidade & $\begin{array}{c}\text { Qualidade e } \\
\text { adequação } \\
\text { do conteúdo }\end{array}$ \\
\hline Qui quadrado (valor) & 18,139 & 28,961 & 0,586 & 1,648 & 30,210 \\
Qui-quadrado (probabilidade) & 0,078 & 0,067 & 0,444 & 0,439 & 0,178 \\
Graus de Liberdade & 11 & 19 & 1 & 2 & 24 \\
GFI - Goodness of Fit & 0,981 & 0,975 & 0,999 & 0,997 & 0,976 \\
CFI - Compartative Fit Index & 0,992 & 0,992 & 1,000 & 1,000 & 0,994 \\
NFI - Normed Fit Index & 0,979 & 0,976 & 0,998 & 0,995 & 0,971 \\
TLI- Tucker-Lewis Index & 0,984 & 0,988 & 1,005 & 1,003 & 0,991 \\
RMR - Root Mean Square Residual & 0,021 & 0,019 & 0,020 & 0,010 & 0,017 \\
RMSEA - R. M. S Error of Aproximation & 0,049 & 0,044 & 0,000 & 0,000 & 0,031 \\
Variância média extraída & 0,890 & 0,910 & 0,800 & 0,850 & 0,910 \\
Alpha de Cronbach & 0,870 & 0,910 & 0,780 & 0,790 & 0,880 \\
\hline \multicolumn{1}{c}{ Índices de ajuste } & Ferramentas & Adequação & Organização & Polo & Satisfação \\
\hline Qui quadrado (valor) & de & do curso & presencial & \\
Qui-quadrado (probabilidade) & 1,234 & 12,822 & 2,071 & 5,178 & 3,980 \\
Graus de Liberdade & 0,540 & 0,250 & 0,355 & 0,159 & 0,552 \\
GFI - Goodness of Fit & 2 & 5 & 2 & 3 & 5 \\
CFI - Compartative Fit Index & 0,998 & 0,983 & 0,996 & 0,993 & 0,995 \\
NFI - Normed Fit Index & 1,000 & 0,982 & 1,000 & 0,997 & 1,000 \\
TLI- Tucker-Lewis Index & 0,997 & 0,971 & 0,994 & 0,993 & 0,996 \\
RMR - Root Mean Square Residual & 1,006 & 0,963 & 0,999 & 0,990 & 1,003 \\
RMSEA - R. M. S Error of Aproximation & 0,100 & 0,022 & 0,008 & 0,013 & 0,007 \\
Variância média extraída & 0,000 & 0,077 & 0,012 & 0,052 & 0,000 \\
Alpha de Cronbach & 0,820 & 0,850 & 0,860 & 0,890 & 0,890 \\
\hline F & 0,790 & 0,792 & 0,850 & 0,870 & 0,890 \\
\hline
\end{tabular}

Fonte: Elaborado pelos autores, 2013.

Após conhecer e validar individualmente cada um dos construtos e suas variáveis formativas buscou-se construir e validar o modelo integrado, que une o modelo de mensuração e o modelo estrutural. A avaliação do modelo teórico foi realizada a partir dos índices de ajuste e da significância estatística dos coeficientes de regressão estimados. Nesse momento cabe uma explicação. Alguns dos construtos inicialmente propostos foram transformados em construtos de segunda ordem visando agrupar em um único construto, fatores que avaliassem tópicos semelhantes. Nesse sentido, criou-se o construto de segunda ordem envolvimento do professor/tutor, o qual foi composto pelos construtos envolvimento do professor e envolvimento do tutor. De forma similar, criou-se o construto de segunda ordem estrutura e organização do sistema e do curso, o qual abrangeu os construtos polo presencial, ferramentas de comunicação, organização do curso e adequação do sistema. A Tabela 4 apresenta os índices de ajuste para o modelo inicial e final.

Após rodado o modelo inicialmente proposto verificou-se que os índices de ajuste não apresentaram valores adequados, por esse motivo procedeu-se a uma série de ajustes. Inicialmente

Tabela 4 - Resultado dos índices de ajuste para o modelo integral

\begin{tabular}{lccccccccc}
\hline $\begin{array}{c}\text { Modelos/Índices } \\
\text { de ajuste }\end{array}$ & $\begin{array}{c}\chi^{2} \\
\text { (valor) }\end{array}$ & $\begin{array}{c}\chi^{2} \\
\text { (probabilidade) }\end{array}$ & $\begin{array}{c}\text { Graus de } \\
\text { liberdade }\end{array}$ & CFI & GFI & NFI & TLI & RMR & RMSEA \\
\hline Modelo inicial & $2.585,08$ & 0,000 & 1406 & 0,870 & 0,747 & 0,756 & 0,863 & 0,088 & 0,056 \\
Modelo final & $2.248,08$ & 0,000 & 1314 & 0,936 & 0,834 & 0,860 & 0,931 & 0,037 & 0,041 \\
\hline Fonte: Elaborado pelos autores, 2013. & & & & & & & &
\end{tabular}


foram excluídas as relações não significativas entre os construtos e inseridas as relações propostas pelo pacote estatístico. Nesse sentido foram excluídas as relações entre os construtos: envolvimento do professor/tutor $\rightarrow$ satisfação dos estudantes de $\mathrm{EaD}$, interação $\rightarrow$ satisfação dos estudantes de $\mathrm{EaD}$, qualidade e adequação do conteúdo $\rightarrow$ satisfação dos estudantes de $\mathrm{EaD}$, estrutura e organização do sistema e do curso $\rightarrow$ interação e incluída a relação entre os construtos envolvimento do professor/ tutor $\rightarrow$ estrutura e organização do sistema e do curso. Como o modelo continuou não apresen-

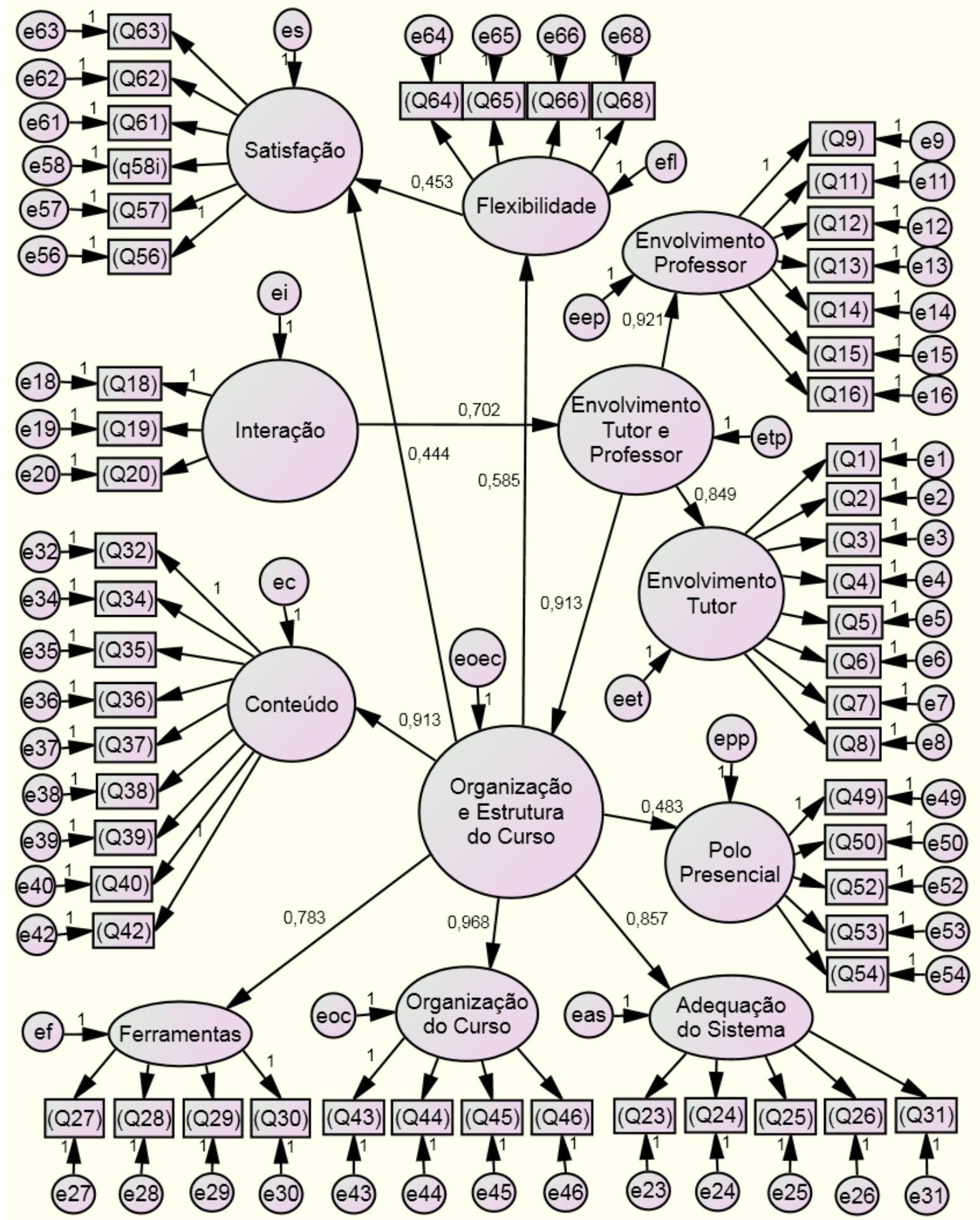

Figura 2 - Modelo final

Fonte: Elaborado pelos autores, 2013. 
Tabela 5 - Coeficientes padronizados e significância das hipóteses

\begin{tabular}{|c|c|c|c|c|c|c|c|}
\hline Hipótese & $\begin{array}{l}\text { Relação entre os } \\
\text { construtos }\end{array}$ & $\begin{array}{c}\text { Coeficientes } \\
\text { padronizados }\end{array}$ & Sig & $\mathrm{Z}$ & \multicolumn{3}{|c|}{ Conclusão } \\
\hline H1 & $\begin{array}{c}\text { Satisfação dos } \\
\text { estudantes de EaD }\end{array}$ & $\leftarrow$ & $\begin{array}{l}\text { Envolvimento } \\
\text { professor/tutor }\end{array}$ & - & 0,303 & 1,029 & Rejeitada \\
\hline $\mathrm{H} 2$ & $\begin{array}{c}\text { Satisfação dos } \\
\text { estudantes de EaD }\end{array}$ & $\leftarrow$ & Interação & - & 0,826 & 0,220 & Rejeitada \\
\hline $\mathrm{H} 3$ & $\begin{array}{l}\text { Envolvimento } \\
\text { professor/tutor }\end{array}$ & $\leftarrow$ & Interação & 0,702 & 0,000 & 5,688 & Confirmada \\
\hline $\mathrm{H} 4$ & $\begin{array}{c}\text { Satisfação dos } \\
\text { estudantes de EaD }\end{array}$ & $\leftarrow$ & Flexibilidade & 0,453 & 0,000 & 7,842 & Confirmada \\
\hline H5 & $\begin{array}{c}\text { Satisfação dos } \\
\text { estudantes de EaD }\end{array}$ & $\leftarrow$ & $\begin{array}{l}\text { Qualidade e adequação } \\
\text { do conteúdo }\end{array}$ & - & 0,506 & 0,665 & Rejeitada \\
\hline H6 & $\begin{array}{c}\text { Satisfação dos } \\
\text { estudantes de EaD }\end{array}$ & $\leftarrow$ & $\begin{array}{c}\text { Estrutura e } \\
\text { Organização do sistema } \\
\text { e do curso } \\
\end{array}$ & 0,444 & 0,000 & 7,384 & Confirmada \\
\hline H7 & $\begin{array}{l}\text { Qualidade e } \\
\text { adequação do } \\
\text { conteúdo }\end{array}$ & $\leftarrow$ & $\begin{array}{c}\text { Estrutura e } \\
\text { Organização do sistema } \\
\text { e do curso }\end{array}$ & 0,913 & 0,000 & 11,524 & Confirmada \\
\hline $\mathrm{H} 8$ & Interação & $\leftarrow$ & $\begin{array}{c}\text { Estrutura e } \\
\text { Organização do sistema } \\
\text { e do curso } \\
\end{array}$ & - & 0,485 & 0,698 & Rejeitada \\
\hline H9 & Flexibilidade & $\leftarrow$ & $\begin{array}{c}\text { Estrutura e } \\
\text { Organização do sistema } \\
\text { e do curso }\end{array}$ & 0,585 & 0,000 & 7,876 & Confirmada \\
\hline
\end{tabular}

Fonte: Elaborado pelos autores, 2013.

tando valores adequados, passou-se a estabelecer correlações entre os erros das questões, desde que os mesmos apresentassem alguma relação teórica justificada pela literatura.

Realizadas tais alterações, verificou-se que o teste qui-quadrado continuou sendo significativo, no entanto passou a apresentar uma razão $\chi^{2} /$ graus de liberdade inferior a $3(2.248,08 / 1314=$ 1,71 ), valor considerado aceitável (KLINE, 1998; HAIR et al., 2009). Os índices RMR $(0,037)$ e RMSEA $(0,041)$ foram adequados, pois apresentaram valores inferiores a 0,10 e 0,08 , respectivamente. Os índices CFI $(0,936)$ e TLI $(0,931)$ também mostraram-se adequados pelo fato de apresentarem valor superior a 0,9 . Já os índices GFI $(0,834)$ e NFI $(0,860)$ não atingiram o valor recomendado pela literatura $(>0,9)$ o que pode ter ocorrido devido ao baixo número de respondentes (416). Entretanto, pelo fato de os outros índices de ajuste terem apresentado valores satisfatórios considerou-se o modelo ajustado. A Figura 2 apresenta o modelo final do estudo.

Com relação ao modelo estrutural, a Tabela 5 apresenta os coeficientes padronizados e a significância das hipóteses.

A primeira hipótese (H1) que afirmava a presença de uma relação positiva entre envolvimento professor/tutor e satisfação dos alunos de $\mathrm{EaD}$ foi rejeitada. No entanto, constatou-se que, devido ao fato de o envolvimento professor/tutor estar associado ao construto estrutura e organização do sistema e do curso, o primeiro construto acaba por exercer uma influência indireta de 0,405 sobre a satisfação dos estudantes de EaD. Conforme Collis (1995), não é a tecnologia em si, mas a correta implementação da tecnologia, resultante do envolvimento e interesse da equipe, que determina os efeitos sobre a aprendizagem. Considerando o efeito indireto do envolvimento do professor/tutor sobre a satisfação e levando em conta que nem todos os professores estão interessados na educação a distância, Sun et al. (2008) sugerem que as instituições devem selecionar os professores cuidadosamente. Para os autores, atrelada à experiência docente, outros critérios devem ser empregados para a seleção de professores para o ensino a distância, tais como: a atitude do professor em relação ao uso do computador e ao uso de tecnologias de rede para a oferta de ensino.

A segunda hipótese proposta (H2) que afirmava a existência de uma relação positiva entre os construtos interação e satisfação dos estudantes de $\mathrm{EaD}$ foi rejeitada por não apresentar significância estatística a um nível de $5 \%$. Apesar de a relação direta sobre a satisfação não ter sido verificada, observa-se que a interação exerce influencia indireta $(0,374)$ através do construto envolvimento do professor/tutor e estrutura e organização do sistema e do curso. O sinal positivo do coeficiente da hipótese $3(\beta=0,702, p=0,000)$ confirma que 
maiores níveis de interação acarretam em um maior envolvimento e interesse de professores e tutores sobre o ensino e a aprendizagem. Para Nelson (1997) apud Paswan e Young (2002), a adoção, por parte dos professores, de uma filosofia de ensino que incentiva e encoraja a participação e discussão, influencia positivamente a avaliação dos estudantes quanto ao nível de dedicação e envolvimento demonstrado pelos professores. De maneira similar, Ali e Ahmad (2011) propõe que um nível mais elevado de interação estudante-estudante e estudante-professor é possível mediante a capacidade dos professores de compreender a diversidade entre os estudantes, encorajar sua participação em discussões e debates e criar um ambiente adequado para o ensino-aprendizagem.

A aceitação da hipótese $4 \quad(\beta=0,453$, $\mathrm{p}=0,000$ ) confirma que a maior flexibilidade proporcionada pelo ensino a distância conduz a maiores níveis de satisfação dos estudantes. Ou seja, a possibilidade de escolher o período de tempo destinado aos estudos, de aprender conforme o próprio ritmo e a possibilidade de melhor organizar as atividades não relacionadas à academia faz com que os estudantes melhorem seu nível de satisfação para com o curso EaD. Segundo Sun et al. (2008), pelo fato de a EaD eliminar as barreiras físicas e temporais, a mesma facilita a aprendizagem construtiva e cooperativa e oportuniza uma interação mais dinâmica o que acaba elevando os níveis de satisfação. Os autores acrescentam que, do ponto de vista operacional, a oportunidade de efetivamente equilibrar trabalho, família e estudos é a primeira vantagem avaliada quando se considera a possibilidade de realização de um curso EaD e, portanto, é fator crítico para a satisfação dos estudantes.

A rejeição da hipótese 5 mostra que, para essa pesquisa, os estudantes não consideraram a qualidade e a adequação do conteúdo como fatores determinantes de seu nível de satisfação. A comprovação da hipótese 6 corrobora o argumento de que uma melhor organização e estrutura do curso conduzem a um maior nível de satisfação dos estudantes (HONG, LAI, HOLTON, 2003; PAECHER, MAIER, MACHER, 2010). A estrutura do sistema desempenha importante papel sobre a percepção do estudante, haja visto que o sistema representa o meio pelo qual os estudantes aprendem e interagem com colegas e professores (OZKAN; KOSELER, 2009). Para Sun et al. (2008), a percepção quanto à real utilidade e facilidade de uso do sistema permite aos estudantes destinar menor esforço no entendimento do sistema e maior esforço no aprendizado do conteúdo o que eleva o nível de satisfação com o aprendizado e com o curso.

A aceitação das hipóteses 7 ( $\beta=0,913$, $\mathrm{p}=0,000)$ e $9(\beta=0,585, \mathrm{p}=0,000)$ demonstra que a estrutura e organização do sistema e do curso além de influenciar diretamente a satisfação dos estudantes de $\mathrm{EaD}$ afeta a percepção dos mesmos quanto à qualidade e adequação do conteúdo e quanto à flexibilidade do curso. Tal resultado sugere que quanto maior o grau de estruturação e adequação do sistema Moodle, das ferramentas de comunicação e do polo presencial e melhor a organização do curso, melhor será a visão do estudante em relação à qualidade do conteúdo desenvolvido nas disciplinas e as vantagens de se realizar um curso a distância. A existência de uma relação positiva entre estrutura e organização do sistema e do curso e interação (H8) não foi confirmada a um nível de significância de $5 \%(\mathrm{p}=0,495)$.

O estabelecimento de uma nova relação positiva entre envolvimento do professor/tutor e estrutura e organização do sistema e do curso, realizado mediante sugestão do pacote estatístico, demonstra que professores e tutores envolvidos com o trabalho tendem a se interessar mais pelo aprendizado dos estudantes e, por esse motivo, buscam trazer para o ambiente de ensino materiais atuais, de boa qualidade e adequados aos objetivos de ensino propostos. Além disso, o envolvimento de professores e tutores contribui para a melhor organização do curso, no sentido de manutenção da disciplina e cumprimento de prazos e carga horária. Para Paswan e Young (2002), uma avaliação positiva quanto ao nível de organização do curso e manutenção de uma estrutura adequada podem contribuir para uma visão favorável quanto ao grau de envolvimento dos professores e tutores.

A confirmação dessas hipóteses vem reafirmar a complexidade de mensuração do construto satisfação, tendo em vista que o mesmo é determinado por um conjunto de fatores que envolve tanto questões de natureza técnica como a flexibilidade e a estrutura e organização do sistema e do curso, quanto questões de natureza subjetiva como o nível de interação com os colegas, professores e tutores e o nível de envolvimento apresentado por tutores e professores. A descoberta de tais questões faz com que as pesquisas sobre satisfação dos estudantes continuem sendo válidas e necessárias, se considerado que as mesmas possibilitam o melhor entendimento acerca dos fatores que podem elevar o nível de satisfação e, por consequência, o nível de aprendizado, pois, como afirmado por Fredericksen (2000) e Levy (2003), estudantes com maiores níveis de satisfação tendem a desenvolver 
níveis consideravelmente mais elevados de aprendizagem se comparados a estudantes com baixo nível de satisfação.

\section{Considerações finals}

A satisfação é um fator primordial para garantir a motivação do discente ao longo da sua formação acadêmica, interferindo no aproveitamento do seu aprendizado e, consequentemente, na competência dos profissionais que serão inseridos no competitivo mercado de trabalho. Tendo em mente tal questão, esse estudo buscou propor e validar um modelo para a compreensão dos fatores determinantes da satisfação dos estudantes de EaD da Universidade Federal de Santa Maria mediante a aplicação de questionários a 416 estudantes durante os meses de outubro, novembro e dezembro de 2012.

$\mathrm{Na}$ etapa descritiva verificou-se que os estudantes avaliaram positivamente todos os construtos pesquisados o que sugere que os mesmos julgam que tanto a instituição de ensino quanto os professores e tutores empenham-se na busca de uma estrutura de comunicação e ensino adequados para o efetivo aprendizado dos estudantes. Além disso, os resultados descritivos demostraram que há pouca variação de resposta entre os estudantes o que sugere que todos os pesquisados tem uma percepção positiva dos fatores investigados e estão, de modo geral, satisfeitos com o curso. A partir da aplicação da modelagem de equações estruturais confirmou-se, em um primeiro momento, a validade de cada um dos construtos testados e a robustez do modelo integrado, dado que todos os índices verificados atingiram os valores recomendados pela literatura. Num segundo momento, analisaram-se as relações causais entre os construtos, sendo confirmadas 5 das 9 hipóteses originalmente propostas. Os resultados ratificam a forte influência positiva da flexibilidade $(45,3 \%)$ e da estrutura e organização do sistema e do curso $(44,4 \%)$ sobre a satisfação dos estudantes de EaD da UFSM.

As hipóteses que relacionavam diretamente o envolvimento do professor/tutor e o nível de interação à satisfação foram rejeitadas. No entanto, tais variáveis exercem influência indireta pelo fato de o envolvimento do professor/tutor estar positivamente relacionado ao construto estrutura e organização do sistema e do curso e, a interação estar positivamente relacionada ao construto envolvimento do professor/tutor. A explicação de $67,2 \%$ da satisfação evidencia a importância de cada um dos construtos validados para a obtenção de melhores níveis de satisfação dos estudantes. A confirmação de boa parte das hipóteses testadas e a obtenção de índices de validade e confiabilidade dentro dos limites aceitos pela literatura valida o modelo como um instrumento de avaliação da satisfação de estudantes de educação a distância.

A partir do estabelecimento de um instrumento para avaliação da satisfação, esse estudo forneceu insights para as instituições de ensino fortalecerem a implementação de seu sistema de educação a distância, estimularem a dinâmica de ensino e aprendizagem e melhorarem os níveis de satisfação dos estudantes. Conforme os resultados obtidos, os fatores envolvimento do professor/ tutor, interação estudante/estudante, estudante/ professor e estudante/tutor, flexibilidade e estrutura e organização do sistema e do curso devem ser considerados para a implementação bem sucedida de um ambiente de educação a distância, tendo em vista que os programas de educação devem proporcionar aos estudantes aquilo que os mesmos valorizam em um processo educacional. De modo geral, os resultados sugerem que entre os desafios futuros das instituições de ensino que ofertam cursos a distância estão a descoberta de novas estratégias e a melhoria do ambiente de aprendizagem online de modo a proporcionar maiores níveis de qualidade de ensino a partir da interação assíncrona e de atividades em grupo.

Embora essa pesquisa tenha representado um esforço cuidadoso e sistêmico para incorporar os fatores relacionados à educação a distância, a mesma apresenta limitações. Como primeira limitação, cita-se o método de coleta de dados via sistema Moodle, o qual apesar de ter se mostrado mais prático não apresentou resultados satisfatórios se considerada a baixa incidência de respondentes. Segunda limitação, apesar de a pesquisa ter englobado boa parcela das variáveis apontadas pela literatura como determinantes da satisfação, tais variáveis não se mostraram suficientes para explicar a totalidade da satisfação dos estudantes. Nesse sentido, pesquisas futuras podem acrescentar outros fatores assinalados pela literatura visando à construção de modelos confiáveis e válidos e a compreensão dos fatores antecedentes da satisfação dos estudantes. Sugere-se ainda a realização de pesquisas longitudinais que possibilitem a avaliação do nível de satisfação ao longo do tempo e de pesquisas com estudantes de EaD de outras instituições de ensino a fim de possibilitar a rea- 
lização de análises comparativas e a verificação da existência de diferenças no relacionamento das variáveis que compõem a satisfação dos estudantes.

\section{Biblografia}

ALI, A.; AHMAD, I. Key factors for determining students' satisfaction in distance learning courses: a study of Allama Iqbal Open University. Contemporary Educational Technology, v. 2, n. 2, p. 118-134, 2011.

ANDERSON, J. C.; GERBING, D. W. Structural equation modeling in practice: a review and recommended two-step approach. Psychological Bulletin, v. 103, p.411-423, 1988.

COLLIS, B. Anticipating the impact of multimedia in education: lessons from the literature. Computers in Adult Education and Training, v.2, n. 2, p. 136149, 1995.

DZIUBAN, C.; MOSKAL, P. Emerging research issues in distributed learning. In: $7^{\mathrm{a}}$ Sloan-C International Conference of Asynchronous Learning Network, 2001.

FREDERICKSEN, E.; PICKETT, A.; SHEA, P.; PELZ, W.; SWAN, K. Student satisfaction and perceived learning with on-line courses: principles and examples from the SUNY learning network. Journal of Asynchronous Learning Networks, v.4, n. 2, p. 7-41, 2000.

GARVER, N. S., MENTZER, J. T. Logistics research methods: employing structural equation modeling to test for construct validity. Journal of Business Logistics, v.20, n.1, p.33-57, 1999.

GIAROLA, E. et al. ambiente virtual de aprendizagem: um estudo sobre a satisfação dos estudantes de administração da Universidade Federal de Lavras. In: Simpósio de Excelência em Gestão e Tecnologia. Anais... Resende, RJ. SEGeT, 2009.

HAIR, J. R. et al. Análise multivariada de dados. $6^{\mathrm{a}}$ ed., Porto Alegre: Bookman, 2009.

HONG, K. S.; LAI, K. W.; HOLTON, D. Students' satisfaction and perceived learning with a web-based course. Educational Technology \& Society, v. 6, n. 1, p.116-124, 2003.

INMAN, E.; KERVIN, M.; MAYES, L. Instructor and student attitudes toward distance learning. Community College Journal of Research and Practice, v. 23, n.6, p. 581-591, 1999.

JOHNSTON, J; KILLION, J; OOMEN, J. Student satisfaction in the virtual classroom. The Internt Journal of Allied Health Sciences and Practice, v. 3, n. 2, 2005.

KLINE, R. B. Principles and practice of structural equation modeling. New York: The Guilford Press, 1998.

LEVY, Y. A study of learners perceived value and satisfaction for implied effectiveness of online learning systems. Dissertation Abstracts International, v. 65, n. 3, 2003.

LIAW, S. S. Investigating students' perceived satisfaction, behavioral intention, and effectiveness of e-learning: a case study of the Blackboard system. Computers \& Education, v. 51, n. 2, p. 864-873, 2008.

LIAW, S. S.; HUANG, H. M. Perceived satisfaction, perceived usefulness and interactive learning

environments as predictors to self-regulation in e-learning environments. Computers \& Education, v. 60 , n. 1, p. 14-24, 2013.

LINDGAARD, G.; DUDEK, C. What is this evasive beast we call user satisfaction? Interacting with Computers, v. 15, n. 3, p. 429-452, 2003.

MACDONALD, C. J.; THOPSON, T. L. Structure, content, delivery, service, and outcomes: quality e-learning in higher education. The international review of research in open and distance learning, v. 6, n. 2, 2005

MOORE, M. G. Three types of interaction. American Journal of Distance Education, v.3. n. 2, p. 1-7. 1989.

MOORE, M. G.; KEARSLEY, G. Distance Education: A Systems View of Online Learning. $3^{\mathrm{a}}$ ed. Cengage Learning, Estados Unidos, 2012.

OLIVEIRA, L. A. B. et al. Modelo para Avaliação da Percepção da Qualidade da Educação a Distância 
Utilizando Estatística Multivariada. In: $36^{\circ}$ Encontro Nacional de Programas de Pós-Graduação. Anais... Rio de Janeiro, 2012.

PAECHTER, M.; MAIER, B.; MACHER, D. Students' expectations of and experiences in e-learning: Their relation to learning achievements and course satisfaction. Computers \& Education, v. 54, n. 1, p. 222-229, 2010.

PASWAN, A.; YOUNG, J. Student evaluation of instructor: A nomelogical investigation using structural equation modeling. Journal of Marketing Education, v.24, n.3, p.193-202, 2002.

PICCOLI, G.; AHMAD, R.; IVES, B. Web-based virtual learning environments: a research framework and a preliminary assessment of effectiveness in basic it skills training. MIS Quarterly,

v. 25 , n. 4 , p. $401-426,2001$.

PITUCH, K. A.; LEE, Y, K. The influence of system characteristics on e-learning use. Computers \& Education, v. 47, n. 2, p. 222-244, 2006.

OZKAN, S.; KOSELER, R. Multi-dimensional students' evaluation of e-learning systems in the higher education context: an empirical investigation. Computers \& Education, v. 53, n. 4, p. 1285-1296, 2009.

SHEE, D. Y.; WANG, Y, S. Multi-criteria evaluation of the web-based e-learning system:

a methodology based on learner satisfaction and its applications. Computers \& Education, v. 50, n. 3, p. 894-905, 2008.

SHER, A. Assessing the relationship of student-instructor and student-student interaction to student learning and satisfaction in web-based online learning environment. Journal of Interactive Online Learning, v. 8, n. 2, p. 102-120, 2009.

SUN, P. C. et al. What drives a successful e-Learning? An empirical investigation of the critical factors influencing learner satisfaction. Computers \& Education, v. 50, p. 1183-1202, 2008.

VALENTA, A., et al. Identifying student attitudes and learning styles in distance education. Journal of Asynchronous Learning Networks, v. 5, n. 2, p. 111127, 2001. 